\title{
Study on One-Dimensional Large Deformation Consolidation of Soil considering Liquid Phase Inertia
}

\author{
Xin-Yu Xie $\mathbb{D}^{1},{ }^{1,2,3}$ Chun-Tai Xu $\mathbb{D},,^{1,2,3}$ Jin-Zhu Li $\mathbb{D}^{2,4}$ Zhong-Jin Wang $\mathbb{D}^{2,4}$ \\ and Wen-Jun Wang $\mathbb{D D}^{2,4}$ \\ ${ }^{1}$ Research Center of Coastal and Urban Geotechnical Engineering, Zhejiang University, Hangzhou 310058, China \\ ${ }^{2}$ Ningbo Institute of Technology, Zhejiang University, Ningbo 315100, China \\ ${ }^{3}$ MOE Key Laboratory of Soft Soils and Geoenvironmental Engineering, Zhejiang University, Hangzhou 310058, China \\ ${ }^{4}$ Ningbo Research Institute, Zhejiang University, Ningbo 315100, China
}

Correspondence should be addressed to Jin-Zhu Li; jinzhu_lee@163.com

Received 6 December 2019; Accepted 5 May 2020; Published 20 May 2020

Academic Editor: Michele Brun

Copyright (c) 2020 Xin-Yu Xie et al. This is an open access article distributed under the Creative Commons Attribution License, which permits unrestricted use, distribution, and reproduction in any medium, provided the original work is properly cited.

\begin{abstract}
Classical consolidation theory ignores the influence of soil liquid phase acceleration. This paper considers the influence of liquid phase acceleration on the stress balance equation during the consolidation of soil, obtains the one-dimensional equation governing quasi-hydrostatic consolidation under large deformation with the consideration of the inertia of the liquid phase, and solves the governing equations by finite element method. The calculation results show that the liquid phase inertia effect of the soil will cause excess pore pressure in the soil, obviously increasing in the initial stage of consolidation, and the self-weight of soil exerts an influence on the excess pore pressure at the later stages of consolidation. The liquid phase inertia effect parameter Dc determines the strength of the liquid phase inertia effect. A larger Dc value results in a larger increase in the excess pore pressure, and the later the liquid phase inertial effect occurs, the longer the duration is. In the large strain consolidation analysis, especially at the initial stage of consolidation, it is necessary to consider the liquid phase inertia effect of the soil.
\end{abstract}

\section{Introduction}

Terzaghi [1] presented his classical consolidation theory of soil in 1925; however, the physical process of soil consolidation shall be further considered for modifying and improving Terzaghi's theory. In the 1960s, many scholars proposed the large strain consolidation theory. Mikasa [2] and Gibson et al. [3] deducted the large strain consolidation control equation with strain and porosity as the control variables, respectively, and considered the impact of soil weight. Pane and Schiffman [4] indicated that equations obtained from different control variables have the same physical principles. The theory proposed by Gibson is widely recognized, and the equation was difficult to solve due to its strong nonlinearity. In light of this, Gibson et al. [5] performed analysis and calculations by assuming that the consolidation coefficient and gravity influence coefficient in the equation were constants.
Many scholars analyze the physical process of soil consolidation under large strain and study the nonlinear problems therein. These studies mainly focus on nonlinear problems related to consolidation and seepage. Davis et al. [6] studied the nonlinear relationship between consolidation coefficients with respect to the void ratio during consolidation and obtained corresponding consolidation curves. Morris [7] analyzed the relationship between the void ratio of soil and effective stress with a semilogarithmic relationship, which is related to strain rate and temperature [8], and proposed the governing equations of nonlinear consolidation theory under large deformation. Moreover, many scholars studied the nonlinear problems of seepage during soil consolidation. Miller and Low [9] discovered the law of seepage with an initial hydraulic gradient and established a relevant seepage model. Hansbo et al. [10] established a nonDarcy seepage model containing an exponential relationship. Based on this model, Li and Xie [11] made relevant 
calculations for solution. Ai and Cang [12] consider the nonaxisymmetric nature of soil penetration, and the calculation of multilayered saturated soil shows that permeability has a significant effect on consolidation. It can be concluded that researchers have been exploring how to elaborate the physical process of soil consolidation under the condition of large deformation.

There are discussions among scholars on how to explain such phenomena from a scientific perspective. Terzaghi et al. [13] use the hydraulic head and pore pressure to obtain a quantity similar in the soil weight to reflect the seepage force as "volume seepage force." Das [14] considered that the seepage force is a force exerted by the seepage on the soil itself. In the deduction of the consolidation theory of soil, many scholars tend to ignore the impact of the inertia of the liquid phase on the stress equilibrium equation. Zhongming et al. [15] studied the seepage force in the total stress equilibrium equation in the application of the effective stress equation. The results showed that the seepage force is not simply related to hydraulic gradient, and the liquid velocity and acceleration should be considered. Ding et al. [16] proposed the kinetic equilibrium equation of consolidation theory under large deformation described by Euler and obtained the soil equilibrium equation with incompressible solid and liquid phases. Ding [17], based on Biot consolidation theory, proposed the effective stress equation and seepage equation applicable to porous media and discussed the seepage force equation considering the influence of seepage velocity and seepage acceleration. Ding [18] further obtained the governing equation of soil consolidation under small strain based on the soil equilibrium equation considering the inertia of the liquid phase.

In this paper, based on Gibson's nonlinear large deformation consolidation theory, by analyzing the impact of the inertia of the liquid phase in the physical process of soil consolidation, the one-dimensional consolidation governing equation with pore pressure as the control variable under large deformation considering the inertia of liquid phase is established. Numerical calculation is adopted to calculate the governing equation, and the development law of excess pore water pressure at the initial stage of soil consolidation is analyzed to demonstrate the impact of the inertia of the liquid phase on the soil consolidation process. The finite element software FlexPDE is employed in the paper to solve the partial differential equation, which is a flexible solution system for partial differential equation, and FlexPDE 7.07 professional version was used in this paper.

\section{Mathematical Model and Deduction of Governing Equations}

2.1. Introduction to the Mathematical Model. Saturated soil can be considered as a porous medium with solid and liquid phases in which drainage and consolidation may occur under applied load. In the continuous medium model, a onedimensional large deformation consolidation equation of soil is deducted with the consideration of the inertia of the liquid phase. For saturated soil with initial thickness $H$, it is permeable at both top and bottom and drainable at both sides. Instantaneously apply a uniformly distributed load $P$ on top of the soil and label the top and bottom of soil in Eulerian coordinates as $x=0$ and $x=X_{G}$. At time $t$, excess pore pressure within the soil is represented with $u$, and the excess pore pressure caused by applied load $Q$ is uniformly distributed along the soil.

During the deduction process, the following assumptions are made: (1) pore-in soil is filled with water, making the soil saturated; (2) both the liquid and solid phases of the soil are incompressible; (3) seepage in the soil complies with Darcy's law, which means that a linear relationship exists between seepage velocity and hydraulic gradient and that the permeability coefficient of Darcy's law remains unchanged during the consolidation process-it is a constant.

2.2. Deduction of the Governing Equation without Considering the Self-Weight of Soil. Analysis is performed on one-dimensional soil in Eulerian coordinates to obtain a one-dimensional quasi-hydrostatic equilibrium equation of soil under large deformation and with incompressible liquid and solid phases by assuming inertia of the solid phase is negligible ((1a) and (1b)):

$$
\begin{aligned}
\frac{\partial \sigma}{\partial x}+\gamma_{m} & =\rho_{f} \frac{\mathrm{D} q}{\mathrm{D} t} \\
\frac{\mathrm{D} q}{\mathrm{D} t} & =\frac{\partial q}{\partial t}+v_{f} \frac{\partial q}{\partial x}
\end{aligned}
$$

where $\sigma$ is the total stress, $\gamma_{m}$ is the gravity of the unit soil, $\rho_{f}$ is the density of the liquid phase (usually water), $q$ is the rate of seepage in the soil, $\mathrm{D} q / \mathrm{D} t$ is the material derivative of the rate of soil seepage, which is the seepage acceleration of soil, and $v_{f}$ is the migration rate of the soil liquid phase, which is used in calculating the material derivative of the seepage rate.

The seepage in soil follows Darcy's law, which establishes the relationship among the seepage rate, hydraulic gradient, and permeability coefficient, and the control equation of seepage in soil is obtained:

$$
q=-\frac{k}{\gamma_{w}} \frac{\partial p}{\partial x},
$$

where $p$ is the pore water pressure, $\partial p / \partial x$ is the pore pressure gradient, also called the hydraulic gradient, and $\gamma_{w}$ is the gravity of the soil liquid phase (generally water). According to the relationship between the excess pore water pressure $(u)$ and the pore water pressure $(p)(3)$, the relationship between the seepage rate and the excess pore water pressure can be obtained (4), where $u$ is the excess pore water pressure.

$$
\begin{aligned}
& p=\gamma_{w} x+u, \\
& q=-\frac{k}{\gamma_{w}}\left(\frac{\partial u}{\partial x}+\gamma_{w}\right) .
\end{aligned}
$$

Substituting equation (4) into equation (1), we can use Darcy's law to express the seepage rate and liquid phase migration rate with excess pore pressure and 
obtain one-dimensional quasi-hydrostatic equilibrium equation of soil under large deformation and with incompressible liquid and solid phases:

$$
\frac{\partial \sigma}{\partial x}=\rho_{f}\left(-\frac{k}{\gamma_{w}} \frac{\partial^{2} u}{\partial x \partial t}-\frac{k^{2}}{\gamma_{w}^{2}}\left(\frac{\partial u}{\partial x}+\gamma_{w}\right) \frac{\partial^{2} u}{\partial x^{2}}\right)-\gamma_{m} .
$$

Integrate total stress with respect to the coordinates to obtain spatial distribution at time $t$. As in practice, conditions of continuity, convergence, and uniform convergence are generally satisfied, and, calculating the partial derivative with respect to time, an expression of the rate of total stress change can be obtained:

$$
\frac{\partial \sigma}{\partial t}=\int_{X_{G}}^{x} \rho_{f}\left(-\frac{k}{\gamma_{w}} \frac{\partial^{3} u}{\partial x \partial^{2} t}-\frac{k^{2}}{\gamma_{w}^{2}} \frac{\partial^{2} u}{\partial x \partial t} \frac{\partial^{2} u}{\partial x^{2}}-\frac{k^{2}}{\gamma_{w}^{2}}\left(\frac{\partial u}{\partial x}+\gamma_{w}\right) \frac{\partial^{3} u}{\partial^{2} x \partial t}\right) \mathrm{d} x+\frac{\partial \gamma_{m}}{\partial t}\left(Z_{G}-x\right) .
$$

Assume the self-weight change of the soil during the consolidation process is negligible and take into consideration the integral of the composite function with a mixed partial derivative. At the bottom surface $x=X_{G}$, since it is assumed to be the drainage surface, the excess pore pressure is always zero, and the consolidation in the neighborhood is completed instantaneously; therefore, the boundary conditions at $x=X_{G}$ are $u=0, \partial u / \partial t=0$, and $\partial p / \partial x=0$. According to the mentioned boundary conditions, expression (6) is calculated and simplified, and an expression of the rate of total stress change can be obtained:

$\frac{\partial \sigma}{\partial t}=\rho_{f}\left[-\frac{k}{\gamma_{w}} \frac{\partial^{2} u}{\partial^{2} t}-\frac{2 k^{2}}{\gamma_{w}^{2}} \frac{\partial^{2} u}{\partial x \partial t} \frac{\partial u}{\partial x}\right]=-\frac{k}{g} \frac{\partial^{2} u}{\partial^{2} t}-\frac{2 k^{2}}{\gamma_{w} g} \frac{\partial^{2} u}{\partial x \partial t} \frac{\partial u}{\partial x}$.

In accordance with the continuity condition in the Gibson equation governing consolidation under large deformation, the continuity equation used by Gibson in one dimension is shown in expression (8), where $\rho$ is the saturation of the soil and $\rho_{w}$ is the density of the water. This continuous (8) can be further calculated to (9), which is the basic equation for large deformation consolidation.

$$
\begin{gathered}
\frac{\partial \rho}{\partial t}+\frac{\partial\left(\rho_{w} q\right)}{\partial x}=0 \\
\frac{1}{1+e} \frac{\partial e}{\partial t}+\frac{\partial q}{\partial x}=0 .
\end{gathered}
$$

Take into consideration constitutive relation of the soil, which means that porosity $e$ is solely related to effective stress $\sigma^{\prime}$, and using the soil seepage equation (2), the consolidation equation of stress can be obtained:

$$
\frac{1}{1+e} \frac{d e}{d \sigma^{\prime}} \frac{\partial \sigma^{\prime}}{\partial t}+\frac{\partial}{\partial x}\left[\frac{k}{\gamma_{w}}\left(\frac{\partial u}{\partial x}+\gamma_{w}\right)\right]=0 .
$$

In accordance with Terzaghi's theory of effective stress (11a) and by calculating partial derivatives with respect to time variable at both sides of (11b), the equivalent relationship between the rate of void ratio with total stress in soil and excess pore pressure is obtained in (12).

$$
\begin{gathered}
\sigma^{\prime}=\sigma-u, \\
\frac{\partial \sigma^{\prime}}{\partial t}=\frac{\partial \sigma}{\partial t}-\frac{\partial u}{\partial t},
\end{gathered}
$$

$$
\frac{1}{1+e} \frac{\partial e}{\partial t}=\frac{1}{1+e} \frac{\mathrm{d} e}{\mathrm{~d} \sigma^{\prime}}\left(\frac{\partial \sigma}{\partial t}-\frac{\partial u}{\partial t}\right)
$$

Combining equation (7), equation (12), and consolidation equation (10), equation (13) is obtained. Further simplification can obtain the large deformation consolidation control equation with the excess pore pressure as the control variable in (14).

$$
\begin{gathered}
\frac{1}{1+e} \frac{\mathrm{d} e}{\mathrm{~d} \sigma^{\prime}}\left(-\frac{k}{g} \frac{\partial^{2} u}{\partial^{2} t}-\frac{2 k^{2}}{\gamma_{w} g} \frac{\partial^{2} u}{\partial x \partial t} \frac{\partial u}{\partial x}-\frac{\partial u}{\partial t}\right) \\
+\frac{\partial}{\partial x}\left[\frac{k}{\gamma_{w}}\left(\frac{\partial u}{\partial x}+\gamma_{w}\right)\right]=0, \\
\frac{\partial u}{\partial t}+\frac{k}{g} \frac{\partial^{2} u}{\partial^{2} t}+\frac{2 k^{2}}{\gamma_{w} g} \frac{\partial^{2} u}{\partial x \partial t} \frac{\partial u}{\partial x}=\frac{k(1+e)}{\gamma_{w}} \frac{\mathrm{d} \sigma^{\prime}}{\mathrm{d} e} \frac{\partial^{2} u}{\partial^{2} x} .
\end{gathered}
$$

Equation (14) is derived under the Euler coordinates because the calculations under the Euler coordinate system need to consider the moving boundary problems, which leads to complicated mathematical processing; thus, a Lagrangian coordinate system is selected for calculating this equation (14). According to the relationship between the Lagrangian and Euler coordinates, the equation of soil consolidation control in the Lagrangian coordinate system is obtained in (16).

$$
\frac{\partial z}{\partial x}=\frac{1+e_{0}}{1+e}
$$

$\frac{\partial u}{\partial t}+\frac{k}{g} \frac{\partial^{2} u}{\partial^{2} t}+\frac{2 k^{2}}{\gamma_{w} g}\left(\frac{1+e_{0}}{1+e}\right)^{2} \frac{\partial^{2} u}{\partial z \partial t} \frac{\partial u}{\partial z}=\frac{\partial}{\partial z}\left(\frac{k\left(1+e_{0}\right)}{\gamma_{w}(1+e)} \frac{\mathrm{d} \sigma^{\prime}}{\mathrm{d} e} \frac{\partial u}{\partial z}\right)$

In Gibson assumptions, coefficient of consolidation $C_{v}$ remains unchanged during soil consolidation (17). The permeability coefficient remains unchanged for further assumptions equation (18). The original governing equation (16) can be simplified to equation (19), which is a one-dimensional equation governing quasi-hydrostatic consolidation under large deformation with self-weight change of soil neglected and with consideration of the inertia of the liquid phase. 


$$
\begin{aligned}
\frac{k(e)}{\gamma_{w}(1+e)} \frac{d \sigma^{\prime}}{d e} & =g(e)=C_{v}=\text { const } \\
\frac{k(e)}{\gamma_{w}(1+e)} & =k(e)=k_{v}=\text { const, } \\
\frac{\partial u}{\partial t}+\frac{k}{g} \frac{\partial^{2} u}{\partial^{2} t}+\frac{2 \gamma_{w} k_{v}^{2}}{g} \frac{\partial^{2} u}{\partial x \partial t} \frac{\partial u}{\partial x} & =C_{v} \frac{\partial^{2} u}{\partial^{2} x} .
\end{aligned}
$$

Simplifying the equation with normalized parameters (20), equation (19) is converted to equation (21).

$$
\begin{aligned}
\frac{k}{g} & =C_{a}, \\
\frac{2 \gamma_{w} k_{v}^{2}}{g} & =C_{f}, \\
\phi & =\frac{u}{P}, \\
Z & =\frac{z}{H}, \\
T & =\frac{C_{v} t}{H^{2}}, \\
\frac{\partial \phi}{\partial T}+\frac{C_{a} C_{v}}{H^{2}} \frac{\partial^{2} \phi}{\partial^{2} T}+\frac{2 C_{a} C_{f} P}{H^{2}} \frac{\partial^{2} \phi}{\partial Z \partial T} \frac{\partial \phi}{\partial Z} & =\frac{\partial^{2} \phi}{\partial^{2} Z},
\end{aligned}
$$

where $P$ is the applied load, $H$ is the thickness of the soil layer, $k$ is the permeability coefficient, $g$ is the acceleration of gravity, $\phi$ is the normalized excess pore pressure, $Z$ is the normalized soil depth, $T$ is the normalized time factor, and $C_{v}$ is the consolidation coefficient.

\subsection{Deduction of the Governing Equation with Consideration} of the Self-Weight Change of Soil. When considering the soil self-weight, the soil continuity equation (9) needs to be appropriately modified. This paper derives from the continuity equation (22) used by Gibson in consideration of the soil self-weight.

$$
\frac{1}{1+e} \frac{\partial e}{\partial t}=\frac{\partial}{\partial x}\left[\frac{k}{\gamma_{w}} \frac{d \sigma^{\prime}}{d e} \frac{\partial e}{\partial z}\right]+\frac{k\left(\gamma_{s}-\gamma_{w}\right)}{\gamma_{w}(1+e)} \frac{\mathrm{d}}{\mathrm{d} e}\left(\frac{\mathrm{d} e}{\mathrm{~d} \sigma^{\prime}}\right) \frac{\partial e}{\partial z} .
$$

In Gibson assumptions, coefficients of both consolidation and gravity of the soil during the consolidation process remain unchanged:

$$
\begin{aligned}
& -\frac{\mathrm{d}}{\mathrm{d} e}\left(\frac{\mathrm{d} e}{\mathrm{~d} \sigma^{\prime}}\right)=\lambda=\text { const }, \\
& \frac{k(1+e)}{\gamma_{w}} \frac{\mathrm{d} \sigma^{\prime}}{\mathrm{d} e}=g(e)=C_{v}=\text { const. }
\end{aligned}
$$

Equation (23) can be integrated to obtain the relationship between excess effective and the ratio of voids, where $\lambda$ is the coefficient of gravity, $e$ is the void ratio, $e_{\infty}$ is the final void ratio, and $e_{0}$ is the initial void ratio. Based on the relationship between the effective stress and the void ratio equation (25), the relationship between the excess pore pressure and the void ratio was obtained by Ding in equation (26), where $Q$ is the applied load.

$$
\begin{aligned}
\sigma^{\prime} & =-\frac{1}{\lambda} \ln \left(\frac{e-e_{\infty}}{e_{0}-e_{\infty}}\right), \\
u & =\left(\gamma_{s}-\gamma_{w}\right) x+\frac{1}{\lambda} \ln \left(\frac{e-e_{\infty}}{e_{0}-e_{\infty}}\right)+Q .
\end{aligned}
$$

Further deriving equation (26) to obtain equation (27) and substituting it into the governing equation (22) simultaneously with (12), (22), and (27), soil control equation (28) can be obtained:

$$
\begin{aligned}
& e=\left(e_{0}-e_{\infty}\right) e^{\lambda u-\lambda\left(\gamma_{s}-\gamma_{w}\right) x-\lambda P}+e_{0} \\
& \frac{\partial u}{\partial t}+\frac{k}{g} \frac{\partial^{2} u}{\partial^{2} t}+\frac{2 k^{2}}{\gamma_{w} g}\left(\frac{1+e_{0}}{1+e}\right)^{2} \frac{\partial^{2} u}{\partial z \partial t} \frac{\partial u}{\partial z} \\
& =\frac{\partial}{\partial z}\left(\frac{k\left(1+e_{0}\right)}{\gamma_{w}(1+e)} \frac{\mathrm{d} \sigma^{\prime}}{\mathrm{d} e} \frac{\partial u}{\partial z}\right)-\lambda\left(\gamma_{s}-\gamma_{w}\right) \frac{\partial u}{\partial z}+\lambda\left(\frac{\partial u}{\partial z}\right)^{2}
\end{aligned}
$$

Applying the normalized parameters (20) and defining new normalized parameters (29), equation (28) can be simplified to equation (30), which is a one-dimensional equation governing quasi-hydrostatic consolidation under large deformation with consideration of self-weight change of soil and inertia of liquid phase, where $D_{c}$ is the liquid phase inertia coefficient, $D_{f}$ is the liquid phase inertia large deformation correction coefficient, $N$ is the soil self-weight gravity coefficient, and $L$ is the applied load gravity coefficient.

$$
\begin{aligned}
N & =\lambda H\left(\gamma_{s}-\gamma_{w}\right), \\
L & =\lambda P, \\
D_{c} & =\frac{C_{a} C_{v}}{H^{2}}, \\
D_{f} & =\frac{2 C_{a} C_{f} P}{H^{2}}, \\
\frac{\partial \phi}{\partial T}+D_{c} \frac{\partial^{2} \phi}{\partial^{2} T}+D_{f} \frac{\partial^{2} \phi}{\partial Z \partial T} \frac{\partial \phi}{\partial Z} & =\frac{\partial^{2} \phi}{\partial^{2} Z}-N \frac{\partial \phi}{\partial Z}+L\left(\frac{\partial \phi}{\partial Z}\right)^{2},
\end{aligned}
$$

2.4. Discussion about the Governing Equation. In the process of deriving the consolidation equation, the influence of liquid phase inertia is especially considered in the quasistatic equilibrium equation of soil. Combined with the relevant assumptions of Gibson's consolidation theory, the one-dimensional equation (31) governing quasi-hydrostatic 
consolidation under large deformation with the consideration of the self-weight change of soil and the inertia of the liquid phase can be obtained.

$$
\frac{\partial \phi}{\partial T}+D_{c} \frac{\partial^{2} \phi}{\partial^{2} T}+D_{f} \frac{\partial^{2} \phi}{\partial Z \partial T} \frac{\partial \phi}{\partial Z}=\frac{\partial^{2} \phi}{\partial^{2} Z}-N \frac{\partial \phi}{\partial Z}+L\left(\frac{\partial \phi}{\partial Z}\right)^{2}
$$

In the case where the thickness of the soil layer is thin or in other conditions where soil can be ignored, the one-dimensional equation (32) can be used governing quasi-hydrostatic consolidation under large deformation with the self-weight change of soil neglected and with the consideration of the inertia of the liquid phase. In equation (32), the influences of the soil self-weight gravity coefficient $N$ and the applied load gravity coefficient $L$ are neglected; only the liquid phase inertial correlation coefficient $D_{c}$ and $D_{f}$ exist. Therefore, equation (32) can calculate the liquid phase inertia effect in large deformation consolidation, but it does not reflect the impact of self-weight.

$$
\frac{\partial \phi}{\partial T}+D_{c} \frac{\partial^{2} \phi}{\partial^{2} T}+D_{f} \frac{\partial^{2} \phi}{\partial Z \partial T} \frac{\partial \phi}{\partial Z}=\frac{\partial^{2} \phi}{\partial^{2} Z} .
$$

Governing equation (32) can be further analyzed. If the large deformation theory is not used and the analysis is performed under the small deformation theory, the time rate of the seepage rate $(\mathrm{D} q / \mathrm{D} t)$ in the original equilibrium equation, that is, equation (1b), can be directly simplified to $\partial q / \partial t$, and its integral formula (6) can also be simplified accordingly. Finally, a one-dimensional consolidation control equation (33) considering liquid phase inertia under the small deformation condition can be obtained:

$$
\frac{\partial \phi}{\partial T}+D_{c} \frac{\partial^{2} \phi}{\partial^{2} T}=\frac{\partial^{2} \phi}{\partial^{2} Z}
$$

On the basis of Terzaghi's consolidation theory, Ding further considered the influence of soil liquid phase and established a one-dimensional quasi-static consolidation model considering inertia of the fluid phase. During his deduction, the self-weight change of soil during consolidation was not considered, and the influence of soil weight on the consolidation process is not reflected in the consolidation control equation. Ding's resulting control equation is consistent with equation (33) under the same normalized variables and parameters. Equation (33) can be further simplified; that is, the liquid phase inertia of the soil can be neglected. At this time, the time change rate of the soil seepage rate in the soil force balance equation (1a) is zero. Based on this derivation, the one-dimensional consolidation control equation (34) under small deformation can be obtained.

$$
\frac{\partial \phi}{\partial T}=\frac{\partial^{2} \phi}{\partial^{2} Z}
$$

The classical consolidation equation (35) relies on the classical one-dimensional consolidation theory presented by Terzaghi in 1925, where $u$ is the excess pore pressure and $z$ is the vertical coordinate under small deformation conditions.
Equation (35) can be simplified by using the same normalized variables in this paper, and consolidation equation (36) in the case of classical small deformation can be obtained. Equation (36) is the same as the deduced result in this paper (see (34)).

$$
\begin{gathered}
\frac{\partial u}{\partial t}=C_{v} \frac{\partial^{2} u}{\partial^{2} z} \\
\frac{\partial \phi}{\partial T}=\frac{\partial^{2} \phi}{\partial^{2} Z}
\end{gathered}
$$

As governing equations (31) and (32) are highly nonlinear, in this paper, finite element software FlexPDE is used for calculations to solve partial differential equations. The calculation results of equations (31)-(34), the equations governing the consolidation of soil under different conditions, are analyzed to study the effect of the inertia of the liquid phase on the consolidation process.

\section{Examples of Calculations and Analyses for Consolidation}

\subsection{Calculation Model}

3.1.1. Parameters of Calculation Examples. In this paper, the calculation example illustrates the excess pore pressure during the process of various types of soil under applied load, which could be referred to soil consolidation. Changes in excess pore pressure during soil consolidation are studied to analyze the effect of the inertia of the liquid phase on consolidation with the following typical calculation parameters of consolidation: soil thickness of 1 meter, applied load on top of the soil of $100 \mathrm{kPa}$, specific gravity of soil particles of $27.5 \mathrm{kN} / \mathrm{m}^{3}$, with water as the liquid phase in the soil, and unit weight of the liquid phase of $9.81 \mathrm{kN} / \mathrm{m}^{3}$. While considering the self-weight of soil, calculations are performed in accordance with the Gibson assumptions of the gravity coefficient of soil $\lambda$ and specific gravity of soil and soil thickness to obtain normalized parameters with respect to the self-weight of the soil: $N=0.3538$ and $L=0.02$.

According to consolidation equation (31), the effect of the inertia of the liquid phase is mainly influenced by inertia parameter of the liquid phase $D_{c}$ and corrected large-deformation parameter $D_{f}$. In light of this, calculations are made with three types of permeability coefficients and compression parameters under three types of typical conditions. In order to highlight the influence of the liquid inertia effect on the soil consolidation process for analysis, the larger permeability coefficient $k$ and the compression modulus $E_{s}$ were selected as the calculation parameters of conditions II and III. The calculation parameters are shown in Table 1. The calculation results are used as the basis for analysis on the effect of the inertia of the liquid phase during consolidation.

3.1.2. Initial and Boundary Conditions. The soil depth is taken as 1 meter, and the top and bottom surfaces are both permeable. The origin of the coordinate is taken on the top 
TABLE 1: Calculation parameters for three typical conditions.

\begin{tabular}{lcccc}
\hline Condition & $k /(\mathrm{m} / \mathrm{s})$ & $E_{s}(\mathrm{MPa})$ & $D_{c}$ & $D_{f}$ \\
\hline I & $1 \times 10^{-9}$ & 2 & $2.078 \times 10^{-17}$ & $2.079 \times 10^{-18}$ \\
II & $1 \times 10^{-3}$ & 40 & $4.158 \times 10^{-4}$ & $2.076 \times 10^{-6}$ \\
III & $2 \times 10^{-3}$ & 40 & $1.639 \times 10^{-3}$ & $8.81 \times 10^{-5}$ \\
\hline
\end{tabular}

surface of the soil, and vertical is the positive direction. Uniform loading is instantaneously applied, and the initial excess pore water pressure is evenly distributed along the depth.

The initial and boundary conditions are as follows:

$$
\left\{\begin{array}{l}
\left.\Phi\right|_{T=0}=1, \\
\left.\Phi\right|_{Z=0}=0, \\
\left.\Phi\right|_{Z=1}=0 .
\end{array}\right.
$$

\subsection{Effect of the Inertia of the Liquid Phase on the Distribution} of Excess Pore Pressure during Consolidation. In this paper, to study the effect of the inertia of the liquid phase on consolidation of the soil, equation (32) is used to obtain the distribution of excess pore pressure at $T=0.001, T=0.002$, and $T=0.005$ under three types of conditions as described in Section 3.1. Calculated distributions of pore pressure are shown in Figures 1-3.

Calculation results shown in Figures 1-3 indicate that as time increases, the excess pore pressure gradually dissipates from the surface of the soil to the interior. These results illustrate that the consolidation of soil is a process that develops over time. It may be considered that an assumed boundary surface exists within the soil. Outside this boundary surface, seepage occurs and excess pore pressure starts to dissipate, whereas within the boundary surface, no seepage occurs and excess pore pressure remains at its initial value. The position of the boundary surface gradually shifts towards inside soil as consolidation develops until it covers the overall soil.

As for the calculation results under conditions II and III, when considering the influence of the inertia of the liquid phase during consolidation, inertia of the liquid phase $\left(D_{c}\right)$ exerts a significant effect on the distribution of excess pore pressure during consolidation, increasing the excess pore pressure near the boundary surface at the beginning of consolidation. This phenomenon was also confirmed in Mandel's experiment, called the Mandel-Cryer effect, which was extensively studied by the academic community. The Mandel-Cryer effect is a typical phenomenon at the initial stage of soil consolidation, which indicates that, under certain conditions, pore pressure in some soil rises rather than dissipates at the initial stage of soil consolidation [19]. Cryer [20] adopted the Biot consolidation theory to solve this problem through the Laplace transform, while Yin and Graham [21] calculated the spherical radial consolidation under uniform pressure and proposed the mechanism explanation. Xie et al. [22] analyzed the changing rule of excess pore water pressure at the initial stage of soil consolidation using the governing equation of large deformation with displacement as the control variable. As a typical phenomenon at the initial stage of soil consolidation, the Mandel-Cryer effect is of great significance to reflect this rule in the consolidation theory of soil.

Calculation results show that, at $T=0.001$, normalized excess pore pressure $\Phi$ outside the boundary surface is increased to 1.03 under condition II $\left(D_{c}=4.158 \times 10^{-4}\right)$ and increased to 1.04 under condition III $\left(D_{c}=1.639 \times 10^{-3}\right)$. This demonstrates that excess pore pressure throughout the outside of the boundary surface is increased under the influence of the inertia of the liquid phase, which is defined as the effect of the inertia of the liquid phase. Comparing the results obtained under conditions II and III shows that a larger control parameter $D_{c}$ results in a larger increase in the excess pore pressure, that is, a more significant effect of the inertia of the liquid phase.

3.3. Effect of the Inertia of the Liquid Phase on the Development of Excess Pore Pressure during Consolidation. By analyzing distribution charts (Figures 1-3) of excess pore pressure at $T=0.001, T=0.002$, and $T=0.005$, it may be observed that the effect of excess pore pressure diminishes with the development of consolidation, for instance, under condition III, at $T=0.001$ and $\Delta \phi_{\max }=0.04$, which is the maximum added value of $\Phi$, at $T=0.002$ and $\Delta \phi_{\max }=0.035$, and at $T=0.005$ and $\Delta \phi_{\max }=0.012$. The results indicate that $\Delta \phi_{\max }$ decreases over time because the increase in excess pore pressure near the drainage surface is due to the liquid phase acceleration caused by increased drainage velocity, and such effect is due to the occurrence of seepage.

To further demonstrate this fact, distribution of the time rate $\left(\partial^{2} \Phi / \partial Z \partial T\right)$ of the change in the excess pore pressure gradient along soil depth $Z$ at $T=0.001, T=0.002$, and $T=0.005$ is plotted and shown in Figure 4. In accordance with Darcy's law equation (38), the seepage acceleration expression is given in equation (39); the excess pore pressure gradient $(\partial \Phi / \partial Z)$ represents seepage velocity in soil, and its time rate of change represents acceleration of seepage.

$$
\begin{gathered}
q=-\frac{k}{\gamma_{w}}\left(\frac{\partial p}{\partial z}+\gamma_{w}\right), \\
\frac{\mathrm{D} q}{\mathrm{D} t}=\frac{\partial q}{\partial t}=-\frac{k}{\gamma_{w}} \frac{\partial^{2} u}{\partial z \partial t} .
\end{gathered}
$$

As consolidation develops, the boundary surface of seepage shifts towards inside soil and the time rate of change of the excess pore pressure gradient at the boundary surface diminishes over time, which means that the seepage velocity at the boundary surface diminishes over time, and, therefore, the effect of the inertia of the liquid phase during consolidation diminishes over time.

The effect of the inertia of the liquid phase significantly influences the development of pore pressure during consolidation. To further understand the effect of parameter $D_{c}$ of the inertia of the liquid phase during consolidation, excess pore pressure is calculated with the governing equation (32) with the self-weight of soil neglected at 

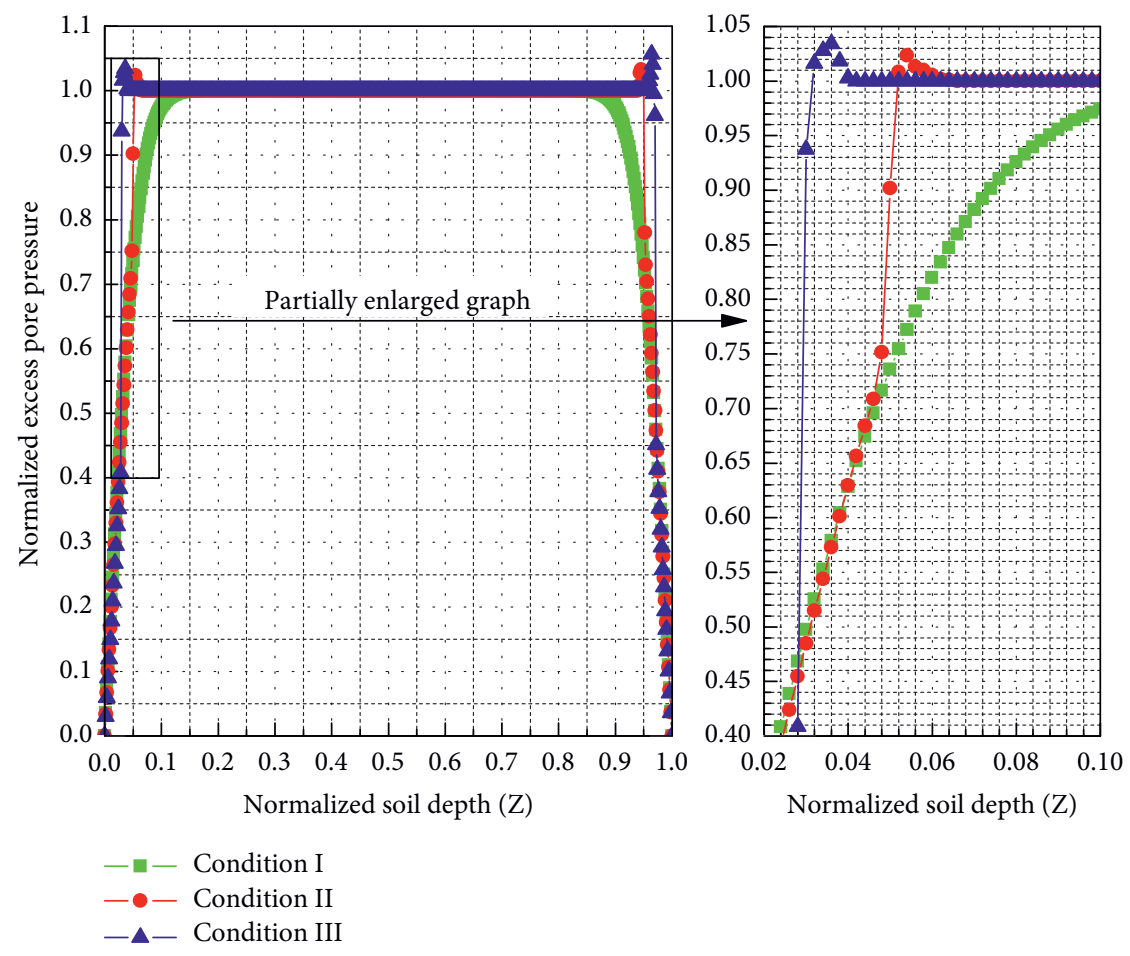

Figure 1: Distribution of normalized excess pore pressure at $T=0.001$.
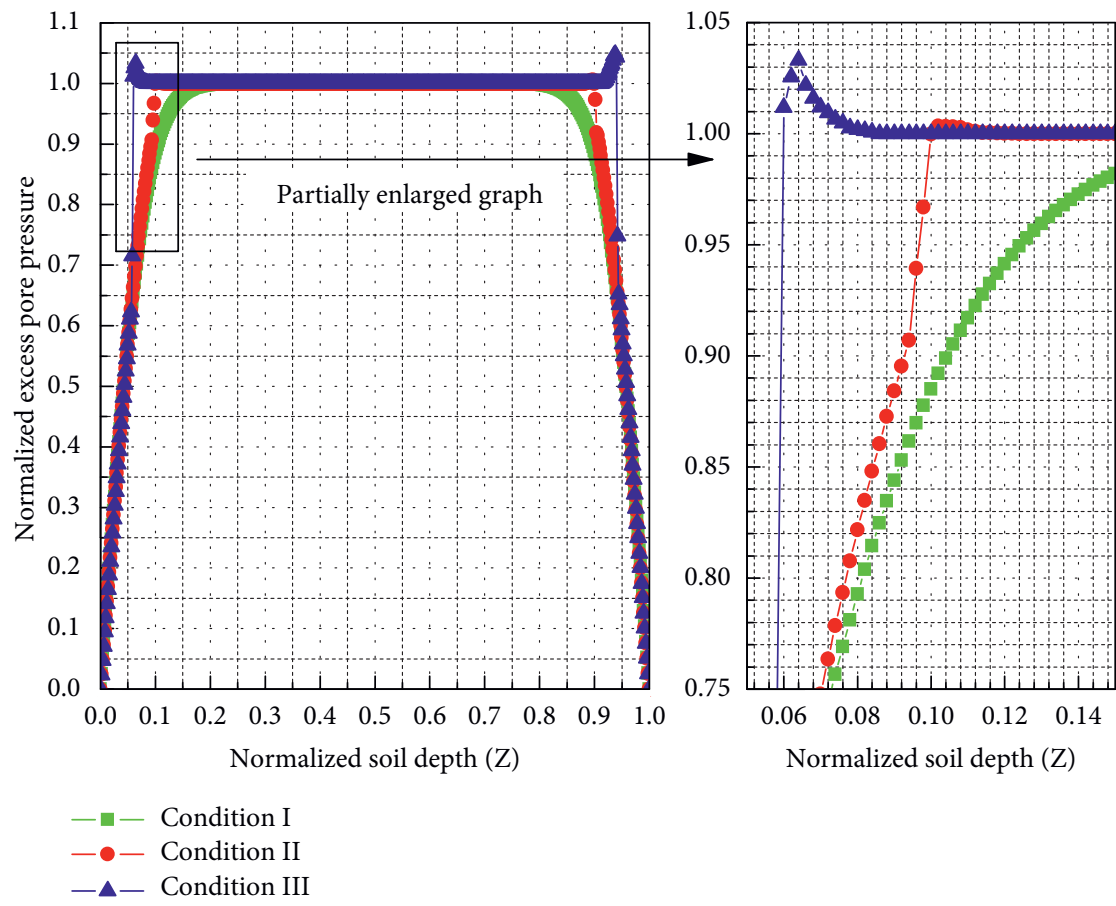

Figure 2: Distribution of normalized excess pore pressure at $T=0.002$.

$Z=0.05$ under three types of conditions. Time-history curves are plotted as shown in Figure 5. At the beginning of consolidation, under two types of conditions with larger $D_{c}$ values, peak excess pore pressure is increased to a certain degree, while the amplitude of the increase and duration increase with increasing $D_{c}$ value, which is defined as the magnifying effect of the inertia of the liquid phase on excess pore pressure during consolidation and proves $D_{c}$ as a parameter representing the effect of the inertia of the liquid phase. 

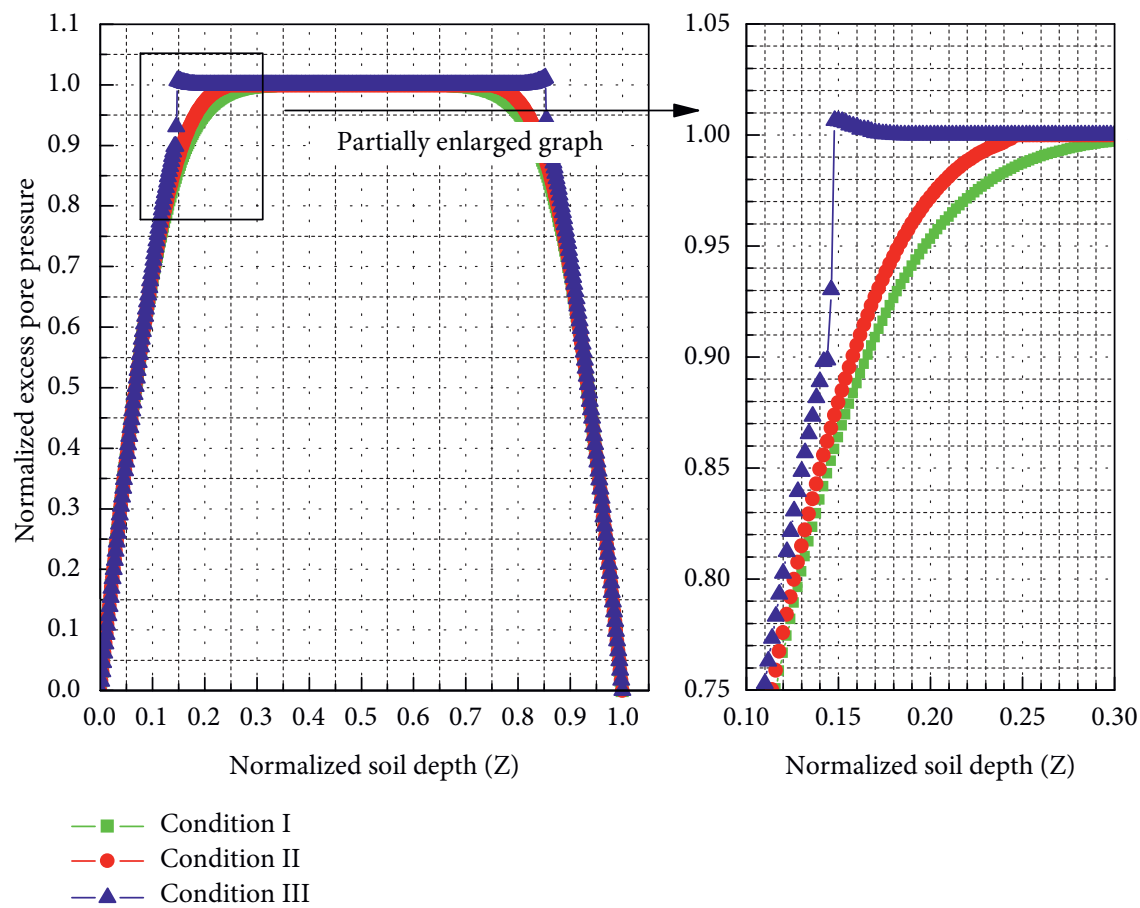

Figure 3: Distribution of normalized excess pore pressure at $T=0.005$.

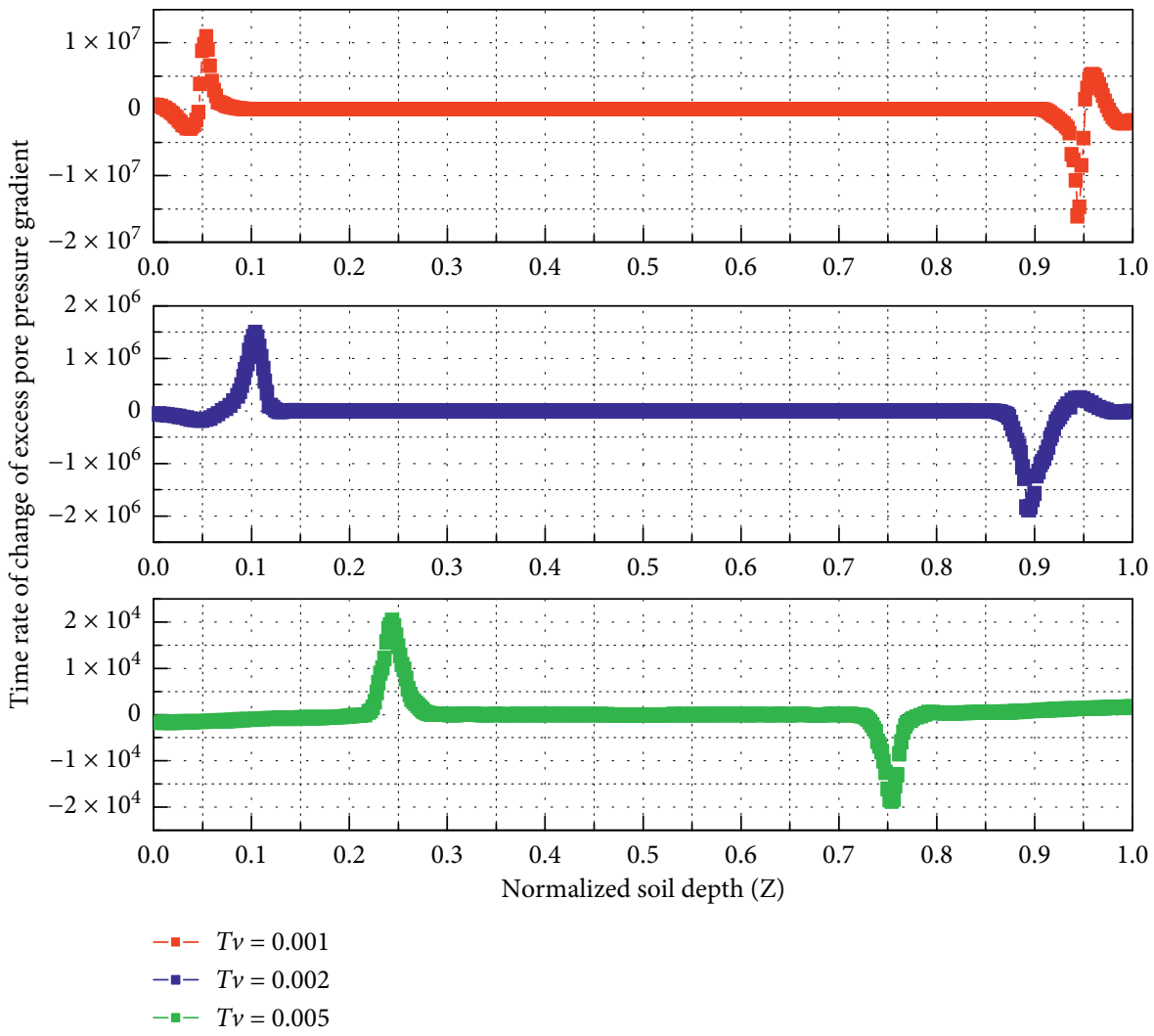

FIgURE 4: Distribution of time rate of change of the excess pore pressure gradient.

Under condition I, the value of $D_{c}$ is minor, which indicates that the effect of the inertia of the liquid phase is negligible. Excess pore pressure is barely increased and is decreased immediately, which is a traditional belief that excess pore pressure dissipates during the development of consolidation. Under condition II, with $D_{c}=4.158 \times 10^{-4}$, 


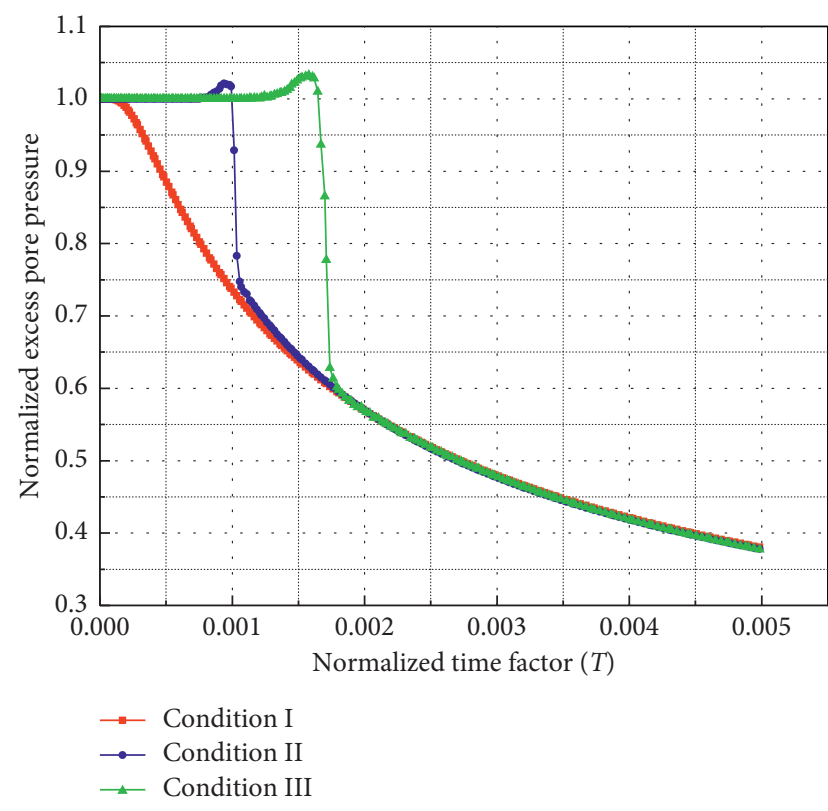

Figure 5: Time-history curves of excess pore pressure at $Z=0.05$ under three conditions.

pore pressure increases at $T=0.0008$ and reaches its maximum value of 1.016 at $T=0.001$. Under condition III, with $D_{c}=1.639 \times 10^{-3}$, pore pressure increases at $T=0.0012$ and reaches its maximum value of 1.032 at $T=0.0016$. Calculation results state that, under both types of conditions, excess pore pressure increases significantly and increases more under condition III compared to that under condition II, and the time that inertia of the liquid phase starts to take effect under condition III lags behind that under condition II with the duration under condition III being longer than that under condition II, which means that a larger $D_{c}$ value will result in a more significant effect of the inertia of the liquid phase.

3.4. Influence of Large Deformation on the Liquid Phase Inertia Effect. To demonstrate the effect of large deformation on the effect of the inertia of the liquid phase, excess pore pressure is calculated with equations (32) and (33) at $Z=0.05$ under condition II, where equation (33) is the governing equation under small deformation theory and equation (32) is the governing equation under large deformation theory. Calculation results shown in Figure 6 indicate that the effect of the inertia of the liquid phase on consolidation of soil calculated under large deformation theory is more significant than that calculated under small deformation theory. The maximum excess pore pressure is increased by 0.0215 when calculated under large deformation theory and increased by 0.02 when calculated under small deformation theory. Calculated pore pressure under large deformation during the subsequent consolidation process is slightly higher than that calculated under small deformation theory. These results state that it is necessary to apply large deformation theory, especially for calculating the maximum excess pore pressure at the beginning of consolidation.
3.5. Effect of the Self-Weight of Soil on the Effect of the Inertia of the Liquid Phase. To demonstrate the effect of the self-weight of soil on the effect of the inertia of the liquid phase, excess pore pressure is calculated with equations (31) and (32) at $Z=0.05$ and $Z=0.95$ under condition II, where equation (32) is the governing equation under large deformation theory with the self-weight of the soil neglected, whereas equation (31) is the governing equation under large deformation theory considering the self-weight of the soil. Calculation results are shown in Figure 7, which indicate that the self-weight of soil exerts a minor influence on the increase in the maximum excess pore pressure due to the effect of the inertia of the liquid phase but exerts a significant influence on the subsequent consolidation process. Without considering the influence of the self-weight of the soil, calculation results show that time-history curves of the excess pore pressure at symmetric positions are coincident because consolidation of soil with two-side drainage with the self-weight of soil neglected can be considered a symmetric process. However, considering the self-weight of the soil, the excess pore pressure at two points in the symmetrical position of the soil will no longer be the same, and the excess pore pressure at the deeper depth will be greater than that at the symmetry point. In Figure 7 , at $Z=0.95$, excess pore pressure obtained with equation (31) is significantly higher than that at $Z=0.05$. According to this analysis, the self-weight of soil exerts a minor influence on the maximum excess pore pressure under the effect of the inertia of the liquid phase but exerts a significant influence on the subsequent consolidation process; therefore, when studying dynamics such as the overall stress in soil at the beginning of consolidation due to inertia of the liquid phase, the self-weight of the soil is negligible.

\section{Discussion}

4.1. Mechanism Interpretation on the Mandel-Cryer Effect. The Mandel-Cryer effect refers to the phenomenon that the excess pore pressure increases instead of dissipating during the initial stage of soil consolidation under certain conditions. Many scholars have studied the seepage force generated by the seepage in the soil. According to the previous analysis, it can be found that, with consideration of the influence of liquid inertia, instead of immediately decreasing, the excess pore pressure near the seepage boundary surface increases to a certain extent and then gradually dissipates during the initial stage of soil consolidation. This phenomenon is the Mandel-Cryer effect. Moreover, it can be seen from the calculation results that this phenomenon becomes even more remarkable as the liquid inertia parameter $D_{c}$ increases.

The phenomenon of the excess pore pressure rising at the early consolidation stage is very similar to the liquefaction in sand or silt soil. Some scholars hold that when the static liquefaction occurs, the excess pore pressure increases significantly under the applied load, which causes the effective stress of the soil to be zero, resulting in the static liquefaction. Sand and silty soils show obvious liquid phase inertia effect. However, the increase range of the excess pore pressure caused by this effect calculated in this paper is less than $10 \%$, so it is a factor leading to static liquefaction, but it is not the 

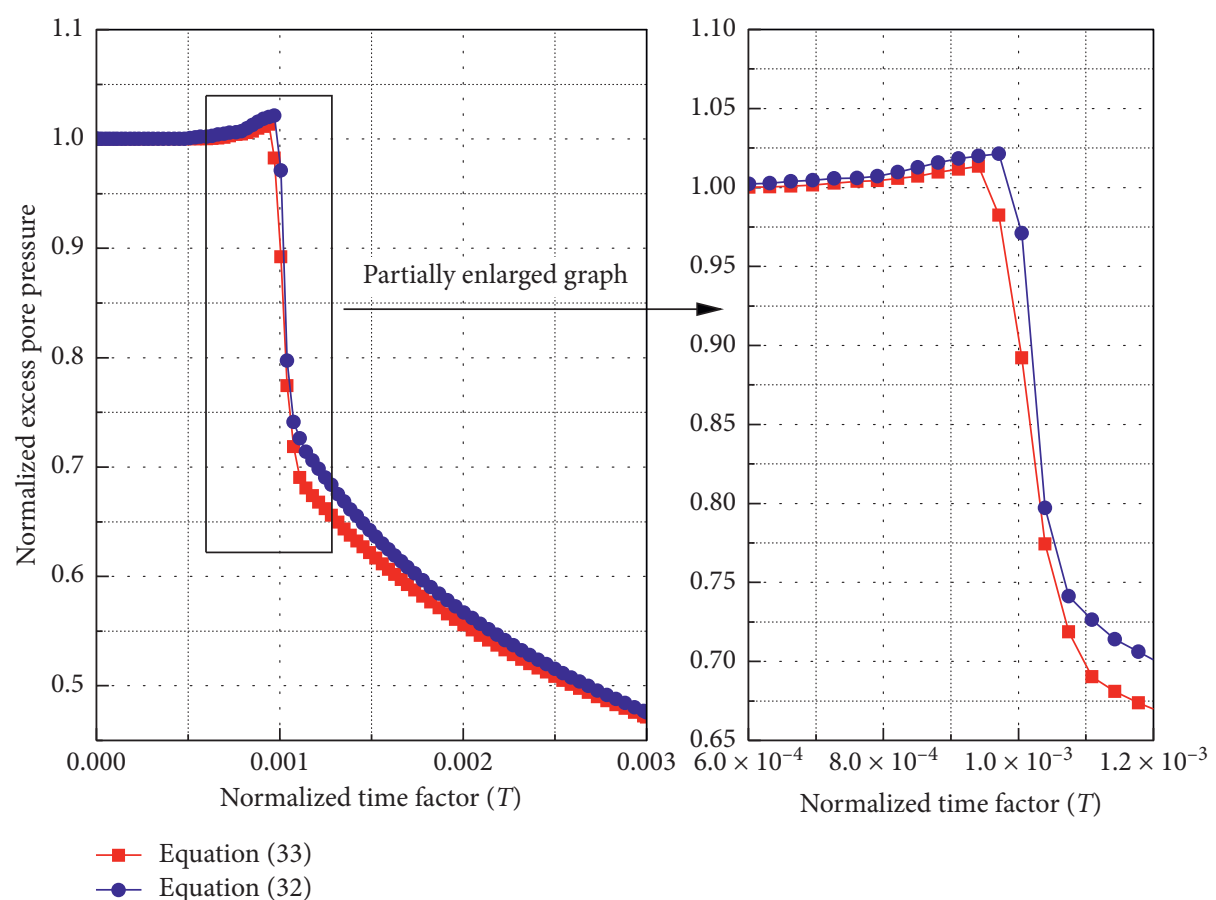

Figure 6: Time-history curves of excess pore pressure at $Z=0.05$ with equations (32) and (33).

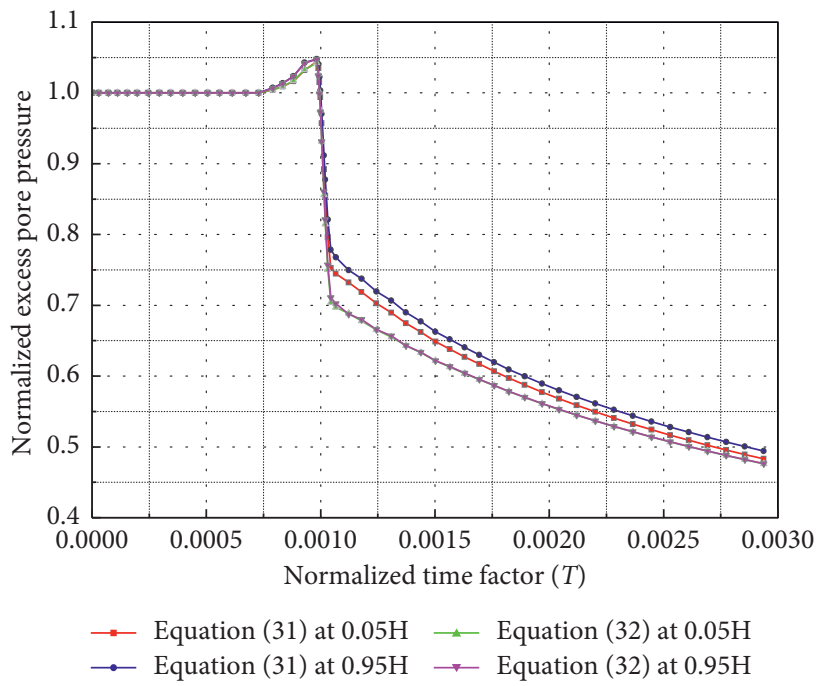

Figure 7: Time-history curves of excess pore pressure at symmetric positions with equations (31) and (32).

main reason. The effect of liquid phase inertia on static liquefaction needs to be further studied.

According to the derivation of the calculation model in this paper, when the rate of change of the applied load at the boundary is assumed to be zero, that is, under the premise that the applied load is instantaneously exerted and remains unchanged, the rate of change in the total stress inside the soil can be obtained as follows:

$\frac{\partial \sigma}{\partial t}=\rho_{f}\left[-\frac{k}{\gamma_{w}} \frac{\partial^{2} u}{\partial^{2} t}-\frac{2 k^{2}}{\gamma_{w}^{2}} \frac{\partial^{2} u}{\partial x \partial t} \frac{\partial u}{\partial x}\right]=-\frac{k}{g} \frac{\partial^{2} u}{\partial^{2} t}-\frac{2 k^{2}}{\gamma_{w} g} \frac{\partial^{2} u}{\partial x \partial t} \frac{\partial u}{\partial x}$.
For the consolidation process of the liquid inertia, it is obvious that the time rate of change of the total stress is controlled by the second-order time rate of change of the excess pore pressure and the pore pressure gradient as well as the time rate of change of the pore pressure gradient when the rate of change of the applied load is zero. Therefore, in the one-dimensional large strain consolidation theory with consideration of the liquid inertia, unlike the finite strain consolidation theory that does not take the liquid inertia into account, the total stress of soil tends to change over time. For the soil at a certain depth $Z$, when the pore pressure of the soil seepage decreases the boundary and reaches depth $Z$, the soil outside $Z$ begins to consolidate, while the soil within $Z$ does not. The seepage in the soil outside $Z$ has liquid inertia, which increases the total stress. However, the soil has not been consolidated within $Z$, so the increased total stress is borne by the internal pore pressure, which increases the excess pore pressure of the soil at the boundary. In short, it could be considered that the liquid inertia leads to an increase in the total stress of the soil. In the case where the soil does not consolidate, the effective stress in the soil is constant and the increase in the total stress generated by the liquid inertia is borne by the internal pore pressure, thus increasing the excess pore pressure.

4.2. Step Phenomenon of Excess Pore Pressure in the Process of Consolidation. For the case where the liquid inertia effect of the soil is more significant, the excess pore pressure will present a step phenomenon. Taking working condition II as an example solving equation (32), the liquid inertial parameter $D_{c}=4.158 \mathrm{e}-4$ is taken to highlight the liquid inertia effect. The soil closer to the drainage surface is selected for analysis, and four representative points at $Z=0.01,0.02$, 


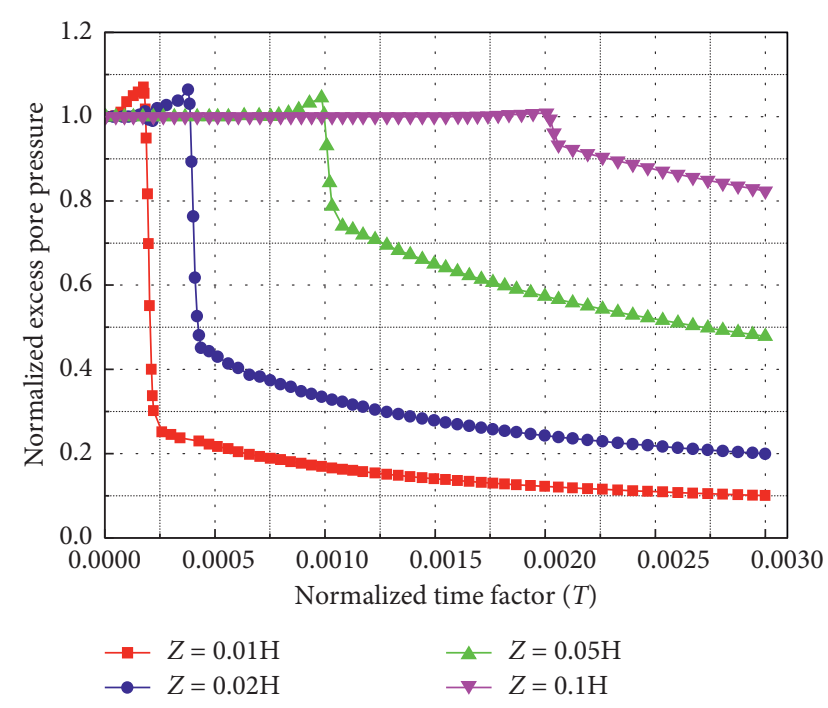

FIGURE 8: Time-history curves of the excess pore pressure at different soil depths.

0.05 , and 0.1 to the drainage surface are analyzed. The timehistory curve of excess pressure is shown in Figure 8.

According to Figure 8, it could be observed that, under a given initial boundary value, the excess pore pressure shows a step phenomenon during the consolidation process. At points $Z=0.01,0.02,0.05$, and 0.1 , the step phenomenon occurrence times are approximately $T=0.0002,0.0004,0.001$, and 0.002 , and a rapid decrease occurs after the excess pore pressure reaches the maximum value; the normalized excess pore pressure reduction values are approximately $0.8,0.6,0.4$, and 0.1 . According to the calculation, it could be found that being farther from the drainage surface results in the step beginning later and the amplitude of the step becoming smaller. For the soil immediately next to the drainage surface, it will start a moment after the initial moment with the initial excess pore pressure as its amplitude under reasonable speculation. These calculation results show that the soil consolidation does not occur instantaneously but rather gradually spreads from the drainage surface to the interior of the soil. Only after it reaches the boundary surface of diffusion does the excess pore pressure of the soil begin to dissipate. The soil at the outer part of the boundary surface has begun to consolidate, and its excess pore pressure value $\Phi_{\text {out }}$ is smaller than the initial excess pore pressure. The soil in the inner part of the boundary surface does not begin to consolidate, and its excess pore pressure value $\Phi_{\text {in }}$ is greater than or equal to the initial excess pore pressure. At a certain depth, the soil changes from the inner part of the boundary surface to the outer part of the boundary surface during the process of pushing the boundary to the interior of the soil. The excess pore pressure is also rapidly reduced from $\Phi_{\text {in }}$, which is higher than or equal to the initial pore pressure, to the excess pore pressure $\Phi_{\text {out }}$ at the outer side of the boundary surface, which is smaller. This kind of sudden drop in the excess pore pressure is the step phenomenon of excess pore pressure in the process of consolidation.

\section{Conclusions}

(1) The effect of the inertia of the liquid phase mainly occurs during the early stage of consolidation of soil and is primarily represented by a slight increase in the excess pore pressure and greater than the initial excess pore pressure when the consolidation starts to occur at the position, which is the Mandel-Cryer effect. The effect of the inertia of the liquid phase is up to its parameter $D_{c}$. A larger $D_{c}$ value means a larger increase in the excess pore pressure, and a more hysteric occurrence of the inertia of the liquid phase indicates a longer duration.

(2) Calculations indicate that the effect of the inertia of the liquid phase under large deformation is more significant than that under small deformation and influences the subsequent consolidation more significantly. The small deformation simplification method underestimates the value of the excess pore pressure during the consolidation process. Larger soil deformation results in a more obvious liquid phase inertia effect and higher error of the small deformation calculation method.

(3) When considering the liquid phase inertia calculation, the self-weight has little effect on the increase in the excess pore pressure during the initial stage of consolidation but exerts a more significant effect on subsequent consolidation. In light of this, the selfweight of soil cannot be ignored when studying consolidation of long duration.

(4) The mechanism of the Mandel-Cryer effect can be explained by the inertial effect of the liquid phase in the soil because the liquid phase inertia effect is obvious during the early stage of consolidation, which leads to an increase in the total stress of the soil. Furthermore, the increase in the total stress is carried by excess pore water pressure, which leads to a slight increase in the excess pore pressure during the early stage of soil consolidation.

(5) After the Mandel-Cryer effect occurs in the initial stage of soil consolidation, the excess pore pressure will have a step phenomenon and decrease rapidly, which is particularly obvious at the edge of the drainage surface. Furthermore, being closer to the drainage surface results in a greater reduction value. Therefore, when calculating the relevant parameters such as pore pressure and effective stress of the soil near the drainage surface, special consideration should be given to the influence of the step phenomenon effect.

\section{Data Availability}

The data used to support the findings of this study are available from the corresponding author upon request. 


\section{Conflicts of Interest}

The authors declare that they have no conflicts of interest.

\section{Acknowledgments}

The authors greatly acknowledge the financial support from the National Natural Science Foundation Projects of China (no. 51708497), Natural Science Foundation of Zhejiang Province (nos. LZ20E080001, LQ19E080009, LY19E080013, and LY20E080001), and the Natural Science Foundation Projects of Ningbo (nos. 2019A610396, 2019C50016, and 2019A610444).

\section{References}

[1] K. Terzaghi, Erdbaumechanik auf bodenphysikalischer Grundlage, Deuticke, Vienna, Austria, 1925.

[2] M. Mikasa, The Consolidation of Soft Clay-A New Consolidation Theory and its application, Kajima Institute, Chofu, Japan, 1965.

[3] R. E. Gibson, G. L. England, and M. J. L. Hussey, "The theory of one-dimensional consolidation of saturated clays," Géotechnique, vol. 17, no. 3, pp. 261-273, 1967.

[4] V. Pane and R. L. Schiffman, "A comparison between two theories of finite strain consolidation," Soils and Foundations, vol. 21, no. 4, pp. 81-84, 1981.

[5] R. E. Gibson, R. L. Schiffman, and K. W. Cargill, "The theory of one-dimensional consolidation of saturated clays. II. Finite nonlinear consolidation of thick homogeneous layers," $\mathrm{Ca}$ nadian Geotechnical Journal, vol. 18, no. 2, pp. 280-293, 1981.

[6] E. H. Davis and G. P. Raymond, "A non-linear theory of consolidation," Géotechnique, vol. 15, no. 2, pp. 161-173, 1965.

[7] P. H. Morris, "Analytical solutions of linear finite-strain onedimensional consolidation," Journal of Geotechnical and Geoenvironmental Engineering, vol. 128, no. 4, pp. 319-326, 2002.

[8] A. Tsutsumi and H. Tanaka, "Combined effects of strain rate and temperature on consolidation behavior of clayey soils," Soils and Foundations, vol. 52, no. 2, pp. 207-215, 2012.

[9] R. J. Miller and P. F. Low, "Threshold Gradient for Water Flow in Clay Systems," Soil Science Society of America Journal, vol. 27, no. 6, pp. 605-609, 1963.

[10] S. Hansbo, "Consolidation of clay with special reference to influence of vertical sand drains," in Proceedings of the Swedish Geotechnical Institute Proceedings, Sweden, 1960.

[11] C. Li and K. Xie, "Large-strain consolidation of soft clay with non-Darcian flow by considering time-dependent load," Chinese Journal of Geotechnical Engineering, vol. 37, no. 6, pp. 1002-1009, 2015.

[12] Z. Y. Ai and N. R. Cang, "Non-axisymmetric Biot consolidation analysis of multi-layered saturated poroelastic materials with anisotropic permeability," Soils and Foundations, vol. 53, no. 3, pp. 408-416, 2013.

[13] K. Terzaghi, R. B. Peck, and G. Mesri, Soil Mechanics in Engineering Practice, John Wiley \& Sons, Hoboken, NJ, USA, 3rd edition, 1996.

[14] B. M. Das, Advanced Soil Mechanics, Taylor\&Francis Group, Oxfordshire, UK, 3rd edition, 2008.

[15] J. Zhongming, L. Fang, X. Xiaohu et al., "Study of calculation methods of acting force of seepage in slope stability analysis," Rock and Soil Mechanics, vol. 36, no. 9, pp. 2478-2486, 2015.
[16] Z. Ding, H. Zhu, and W. Ding, "A strict form of continuity condition in large strain consolidation theory," Journal of Tongji University (Natural Science), vol. 37, no. 4, pp. 471-474, 2009.

[17] Z. Ding, "Mechanical fundamentals of seepage force concept and its generalization," Chinese Journal of Geotechnical Engineering, vol. 39, no. 11, pp. 2088-2101, 2017.

[18] Z. Ding, "One-dimensional quasi-static consolidation model considering inertia of fluid phase," Chinese Journal of Theoretical and Applied Mechanics, vol. 50, no. 4, pp. 908-927, 2018.

[19] R. E. Gibson, A. Gorbeth, and R. L. Schiffman, "On Cryer's problem with large displacements and variable permeability," Géotechnique, vol. 40, no. 6, pp. 627-631, 1990.

[20] C. W. Cryer, "A comparison of the three-dimensional consolidation theories of biot and terzaghi," The Quarterly Journal of Mechanics and Applied Mathematics, vol. 16, no. 4, pp. 401-412, 1963.

[21] J.-H. Yin and J. Graham, "Equivalent times and one-dimensional elastic viscoplastic modelling of time-dependent stress-strain behaviour of clays," Canadian Geotechnical Journal, vol. 31, no. 1, pp. 42-52, 1994.

[22] X. Xie, Y. Liu, and Q. Pan, "Effects of variable permeability on one-dimensional large strain consolidation," Chinese Journal of Geotechnical Engineering, vol. 22, no. 4, pp. 509-511, 2000. 